Internat. J. Math. \& Math. Sci.

Vol. 24, No. 8 (2000) 505-510

S0161171200010310

(C) Hindawi Publishing Corp.

\title{
AN ASYMPTOTIC EXPANSION FOR A RATIO OF PRODUCTS OF GAMMA FUNCTIONS
}

\author{
WOLFGANG BÜHRING
}

(Received 7 April 2000)

\begin{abstract}
An asymptotic expansion of a ratio of products of gamma functions is derived. It generalizes a formula which was stated by Dingle, first proved by Paris, and recently reconsidered by Olver.
\end{abstract}

Keywords and phrases. Gamma function, asymptotic expansion.

2000 Mathematics Subject Classification. Primary 33B15.

1. Introduction. Our starting point is the Gaussian hypergeometric function $F(a, b ; c ; z)$ and its series representation

$$
\frac{1}{\Gamma(c)} F(a, b ; c ; z)=\sum_{n=0}^{\infty} \frac{(a)_{n}(b)_{n}}{\Gamma(c+n) n !} z^{n}, \quad|z|<1,
$$

which here is written in terms of Pochhammer symbols

$$
(x)_{n}=x(x+1) \cdots(x+n-1)=\frac{\Gamma(x+n)}{\Gamma(x)} .
$$

The hypergeometric series appears as one solution of the Gaussian (or hypergeometric) differential equation, which is characterized by its three regular singular points at $z=0,1, \infty$. The local series solutions at zero and 1 of this differential equation are connected by the continuation formula [1],

$$
\begin{aligned}
\frac{1}{\Gamma(c)} F(a, b ; c ; z)= & \frac{\Gamma(c-a-b)}{\Gamma(c-a) \Gamma(c-b)} F(a, b ; 1+a+b-c ; 1-z) \\
& +\frac{\Gamma(a+b-c)}{\Gamma(a) \Gamma(b)}(1-z)^{c-a-b} F(c-a, c-b ; 1+c-a-b ; 1-z), \\
& (|\arg (1-z)|<\pi) .
\end{aligned}
$$

Here we want to show that (1.3) implies an interesting asymptotic expansion for a ratio of products of gamma functions, of which only a special case was known before.

By applying the method of Darboux [5, 9] to (1.3), we derive in Section 2 the formula in question. The behaviour of this and a related formula is discussed in Section 3 and illustrated by a few numerical examples. 
2. Derivation of an asymptotic expansion for a ratio of products of gamma functions. It is well known that the asymptotic behaviour of the Taylor series coefficients contains information about the nearest singular point of the expanded function [4, 5, 9]. (An enlightening more recent paper [3] gives some deeper insight into the interplay between coefficient asymptotics and the behaviour of the expanded function in situations more general than needed for our purpose.) In this respect we want to analyze the continuation formula (1.3). The second term, $R$, on the right-hand side, which may be written as

$$
R=\frac{\Gamma(a+b-c) \Gamma(1+c-a-b)}{\Gamma(a) \Gamma(b)} \sum_{m=0}^{\infty} \frac{(c-a)_{m}(c-b)_{m}}{\Gamma(1+c-a-b+m) m !}(1-z)^{c-a-b+m}
$$

gives the singular expansion of the function around $z=1$, which is the singularity closest to the origin. By means of the binomial theorem in its hypergeometric-seriesform, we may expand the power factor

$$
(1-z)^{c-a-b+m}=\sum_{n=0}^{\infty} \frac{\Gamma(a+b-c-m+n)}{\Gamma(a+b-c-m) n !} z^{n} .
$$

Interchanging the order of summation and simplifying by means of the reflection formula of the gamma function, we arrive at

$$
R=\frac{1}{\Gamma(a) \Gamma(b)} \sum_{n=0}^{\infty} \sum_{m=0}^{\infty}(-1)^{m} \frac{(c-a)_{m}(c-b)_{m}}{m !} \frac{\Gamma(a+b-c-m+n)}{n !} z^{n} .
$$

This is to be compared with the left-hand side, $L$, of (1.3), which is

$$
L=\frac{1}{\Gamma(a) \Gamma(b)} \sum_{n=0}^{\infty} \frac{\Gamma(a+n) \Gamma(b+n)}{\Gamma(c+n) n !} z^{n} .
$$

Comparison of the coefficients of these two power series, which according to Darboux [5] and Schäfke and Schmidt [9] should agree asymptotically as $n \rightarrow \infty$, then yields

$$
\begin{aligned}
\frac{\Gamma(a+n) \Gamma(b+n)}{\Gamma(c+n)}= & \sum_{m=0}^{M}(-1)^{m} \frac{(c-a)_{m}(c-b)_{m}}{m !} \Gamma(a+b-c-m+n) \\
& +O(\Gamma(a+b-c-M-1+n)) .
\end{aligned}
$$

By means of

$$
O(\Gamma(a+b-c-M-1+n))=\Gamma(a+b-c+n) O\left(n^{-M-1}\right)
$$


and the reflection formula of the gamma function, the relevant formula (2.5) may also be written as

$$
\frac{\Gamma(a+n) \Gamma(b+n)}{\Gamma(c+n) \Gamma(a+b-c+n)}=1+\sum_{m=1}^{M} \frac{(c-a)_{m}(c-b)_{m}}{m !(1+c-a-b-n)_{m}}+O\left(n^{-M-1}\right) .
$$

The asymptotic expansion for a ratio of products of gamma functions in this form (2.7) or the other (2.5) seems to be new. It is only the special case when $c=1$ which is known. This special case was stated by Dingle [2], first proved by Paris [8], and reconsidered recently by Olver [6], who has found a simple direct proof. His proof, as well as the proof of Paris, can be adapted easily to the more general case when $c$ is different from 1. Still another proof is available [7] which includes an integral representation of the remainder term. Our derivation of (2.5) or (2.7) is significantly different from all the earlier proofs of the case when $c=1$.

3. Discussion and numerical examples. We now want to discuss our result in the form (2.7). First we observe that the substitution $c \rightarrow a+b-c$ leads to the related formula

$$
\frac{\Gamma(a+n) \Gamma(b+n)}{\Gamma(c+n) \Gamma(a+b-c+n)}=1+\sum_{m=1}^{M} \frac{(a-c)_{m}(b-c)_{m}}{m !(1-c-n)_{m}}+O\left(n^{-M-1}\right) .
$$

Which of (2.7) or (3.1) is more advantageous numerically depends on the values of the parameters, and in this respect the two formulas complement each other.

For finite $n$ and $M \rightarrow \infty$ the series on the right-hand side of (2.7) converges if $\operatorname{Re}(1-c-n)>0$. The same is true for (3.1) if $\operatorname{Re}(1+c-a-b-n)>0$. Then, in both cases, the Gaussian summation formula yields

$$
\frac{\Gamma(1-c-n) \Gamma(1+c-a-b-n)}{\Gamma(1-a-n) \Gamma(1-b-n)}
$$

which, by means of the reflection formula of the gamma function, is seen to be equal to

$$
\frac{\Gamma(a+n) \Gamma(b+n)}{\Gamma(c+n) \Gamma(a+b-c+n)} \frac{\sin (\pi[a+n]) \sin (\pi[b+n])}{\sin (\pi[c+n]) \sin (\pi[a+b-c+n])} .
$$

Otherwise (2.5), (2.7), and (3.1) are divergent asymptotic expansions as $n \rightarrow \infty$.

Although in our derivation $n$ is a sufficiently large positive integer, the asymptotic expansions (2.5), (2.7), and (3.1) are expected to be valid in a certain sector of the complex $n$-plane, and in fact, the proofs of Paris [8] and of Olver [7] apply to complex values of $n$. 
TABLE 3.1. Values of the right-hand sides of (2.7) and (3.1) for the parameters $a=-11.7, b=-11.2, c=-11.4$.

\begin{tabular}{clrc}
\hline & $M$ & right-hand side of $(2.7)$ & right-hand side of (3.1) \\
\hline$n=10$ & 1 & 0.976000 & 0.975000 \\
& 2 & 0.972434 & 0.971912 \\
3 & 0.971341 & 0.971037 \\
4 & 0.970882 & 0.970687 \\
5 & 0.970651 & 0.970517 \\
6 & 0.970520 & 0.970423 \\
7 & 0.970440 & 0.970367 \\
8 & 0.970388 & 0.970331 \\
9 & 0.970352 & 0.970307 \\
10 & 0.970326 & 0.970290 \\
\hline exact value of $(2.7)$ or $(3.1): 1.94045281$ & \\
exact value of $(3.3): 0.97022640$ & \\
\hline 1 & 1.008000 & 1.007895 \\
2 & 1.007360 & 1.007392 \\
3 & 1.007521 & 1.007504 \\
4 & 1.007438 & 1.007452 \\
5 & 1.007515 & 1.007497 \\
6 & 1.007385 & 1.007426 \\
7 & 1.007839 & 1.007650 \\
9 & 1.002201 & 1.005398 \\
\hline exact value of $(2.7)$ & 0.965891 \\
exact value of $(3.3): 0.50373645$ & 0.740024 \\
\hline
\end{tabular}

If the series in (2.7) or (3.1) converge, their sums are equal to (3.3), which generally (if neither $c-a$ nor $c-b$ is equal to an integer) is different from the left-hand side of (2.7) or (3.1). Therefore (2.7) and (3.1) can be valid only in the half-planes in which the series do not converge. This means that (2.7) is an asymptotic expansion as $n \rightarrow \infty$ in the half-plane $\operatorname{Re}(c-1+n) \geq 0$, and (3.1) is an asymptotic expansion as $n \rightarrow \infty$ in the half-plane $\operatorname{Re}(a+b-c-1+n) \geq 0$. Otherwise the series on the right-hand sides represent a different function, namely (3.3).

A few numerical examples may serve for demonstration of these facts. In Table 3.1, the series converge to (3.3) for $n=10$, and therefore (2.7) and (3.1) are not valid. For $n=20$, on the other hand, the series diverge and so (2.7) and (3.1) hold. The transition between the two regions is at the line $\operatorname{Re}(n)=12.4$ in case of $(2.7)$ or $\operatorname{Re}(n)=12.5$ in case of (3.1). In Table 3.2, we see convergence for $n=-15$ and divergence for $n=-5$, the transition between the two regions being at the line $\operatorname{Re}(n)=-10.4$ in case of (2.7) or $\operatorname{Re}(n)=-10.5$ in case of (3.1). 
TABLE 3.2. Values of the right-hand sides of (2.7) or (3.1) for the parameters $a=11.7, b=11.2, c=11.4$.

\begin{tabular}{|c|c|c|c|}
\hline & $M$ & right-hand side of (2.7) & right-hand side of (3.1) \\
\hline \multirow[t]{12}{*}{$n=-15$} & 1 & 0.986667 & 0.986957 \\
\hline & 2 & 0.985648 & 0.985745 \\
\hline & 3 & 0.985453 & 0.985492 \\
\hline & 4 & 0.985397 & 0.985415 \\
\hline & 5 & 0.985376 & 0.985386 \\
\hline & 6 & 0.985368 & 0.985373 \\
\hline & 7 & 0.985363 & 0.985367 \\
\hline & 8 & 0.985361 & 0.985363 \\
\hline & 9 & 0.985360 & 0.985361 \\
\hline & 10 & 0.985359 & 0.985360 \\
\hline & \multicolumn{3}{|c|}{ exact value of (2.7) or (3.1): 1.97071532} \\
\hline & \multicolumn{3}{|c|}{ exact value of (3.3): 0.98535766} \\
\hline \multirow[t]{12}{*}{$n=-5$} & 1 & 1.010909 & 1.011111 \\
\hline & 2 & 1.009891 & 1.009798 \\
\hline & 3 & $1.010254 \longleftarrow$ & $1.010331 \longleftarrow$ \\
\hline & 4 & $1.009940 \longleftarrow$ & $1.009818 \longleftarrow$ \\
\hline & 5 & 1.010589 & 1.011015 \\
\hline & 6 & 1.005300 & 0.998322 \\
\hline & 7 & 0.951894 & 0.887892 \\
\hline & 8 & 0.737202 & 0.459630 \\
\hline & 9 & 0.134729 & -0.725230 \\
\hline & 10 & -1.243041 & -3.418810 \\
\hline & exa & $(2.7)$ or $(3.1): 1.01011438$ & \\
\hline & exa & $f(3.3): 0.50505719$ & \\
\hline
\end{tabular}

\section{REFERENCES}

[1] M. Abramowitz and I. A. Stegun, (Contributors), Handbook of Mathematical Functions, with Formulas, Graphs, and Mathematical Tables, Third printing with corrections. National Bureau of Standards Applied Mathematics Series, no. 55, Superintendent of Documents, U.S. Government Printing Office, Washington, D.C., 1965. MR 31\#1400.

[2] R. B. Dingle, Asymptotic Expansions: their Derivation and Interpretation, Academic Press [A subsidiary of Harcourt Brace Jovanovich, Publishers], London, New York, 1973. MR 58\#17673. Zbl 279.41030.

[3] P. Flajolet and A. Odlyzko, Singularity analysis of generating functions, SIAM J. Discrete Math. 3 (1990), no. 2, 216-240. MR 90m:05012. Zbl 712.05004.

[4] P. M. Morse and H. Feshbach, Methods of Theoretical Physics, vol. 1, McGraw-Hill Book Co., Inc., New York, Toronto, London, 1953, pp. 1-997. MR 15,583h. Zbl 051.40603.

[5] F. W. J. Olver, Asymptotics and Special Functions, Academic Press [A subsidiary of Harcourt Brace Jovanovich, Publishers], New York, London, 1974, Computer Science and Applied Mathematics. MR 55\#8655. Zbl 303.41035. 
[6] _ Asymptotic expansions of the coefficients in asymptotic series solutions of linear differential equations, Methods Appl. Anal. 1 (1994), no. 1, 1-13. MR 95d:34088. Zbl 838.34071.

[7] _ On an asymptotic expansion of a ratio of gamma functions, Proc. Roy. Irish Acad. Sect. A 95 (1995), no. 1, 5-9. MR 97e:33006. Zbl 848.33001.

[8] R. B. Paris, Smoothing of the Stokes phenomenon using Mellin-Barnes integrals, J. Comput. Appl. Math. 41 (1992), no. 1-2, 117-133, Asymptotic methods in analysis and combinatorics. MR 94e:41042. Zbl 772.33015.

[9] R. Schäfke and D. Schmidt, The connection problem for general linear ordinary differential equations at two regular singular points with applications in the theory of special functions, SIAM J. Math. Anal. 11 (1980), no. 5, 848-862. MR 82a:34010a. Zbl 442.34008 .

Wolfgang BÜHring: Physikalisches Institut, Universität HeIDElberg, PhilosopheNWEG 12, 69120 HEIDELBERG, GERMANY

E-mail address: buehring@physi .uni-heide1berg.de 


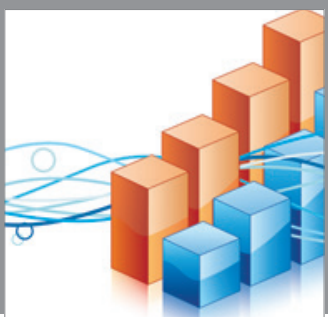

Advances in

Operations Research

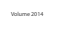

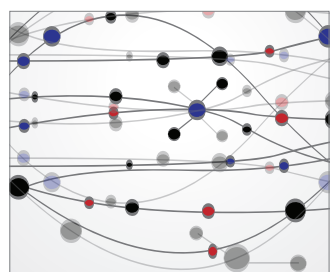

\section{The Scientific} World Journal
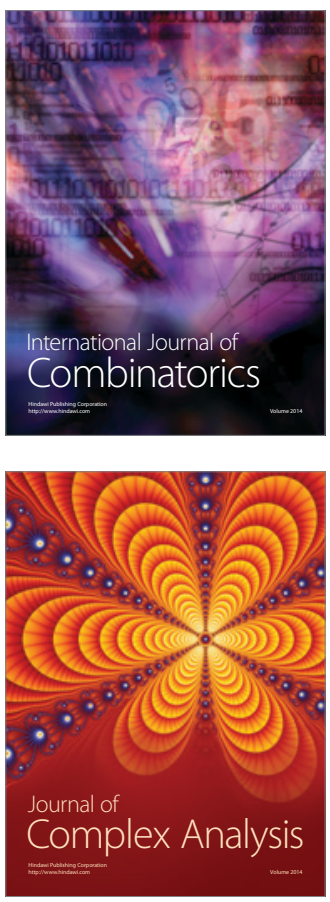

International Journal of

Mathematics and

Mathematical

Sciences
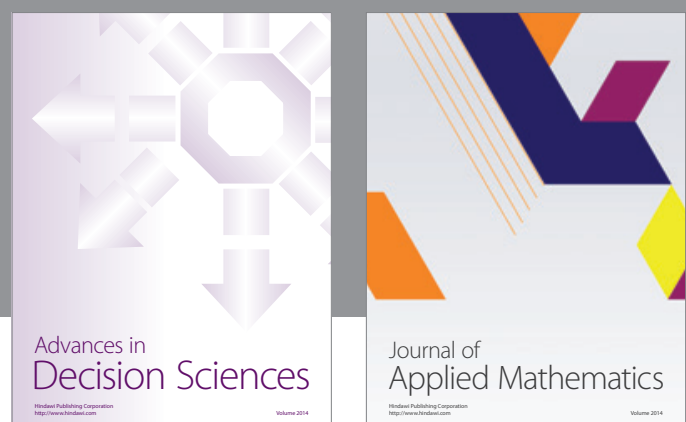

Journal of

Applied Mathematics
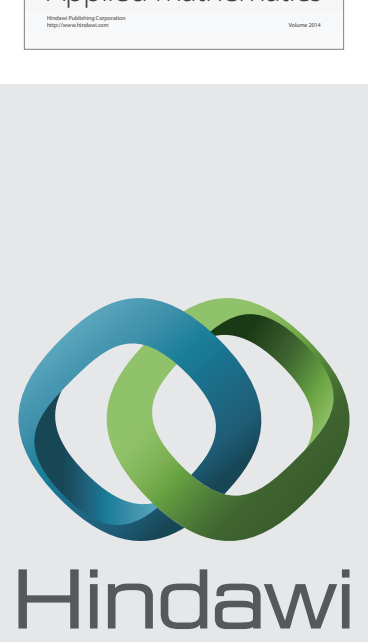

Submit your manuscripts at http://www.hindawi.com
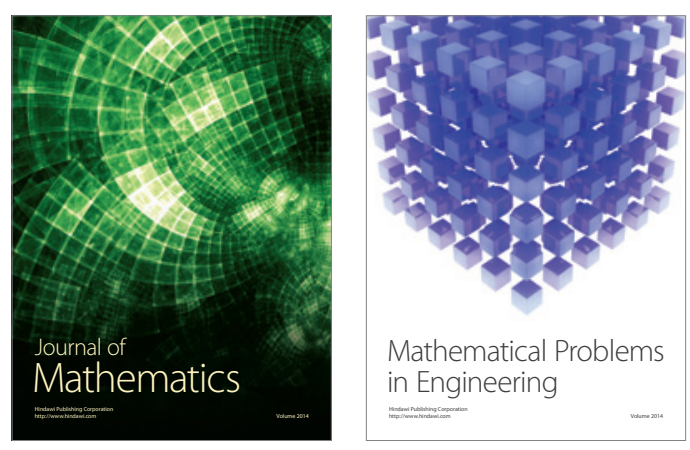

Mathematical Problems in Engineering
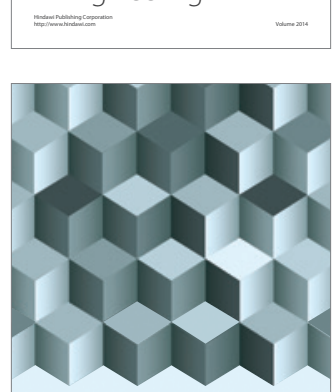

Journal of

Function Spaces
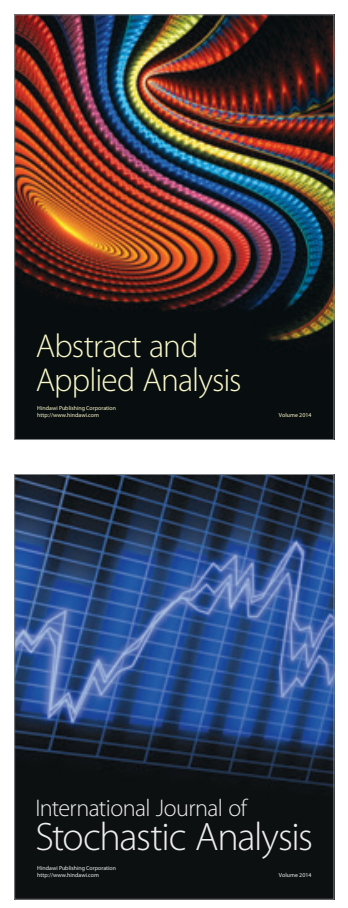

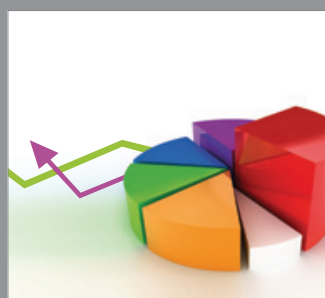

ournal of

Probability and Statistics

Promensencen
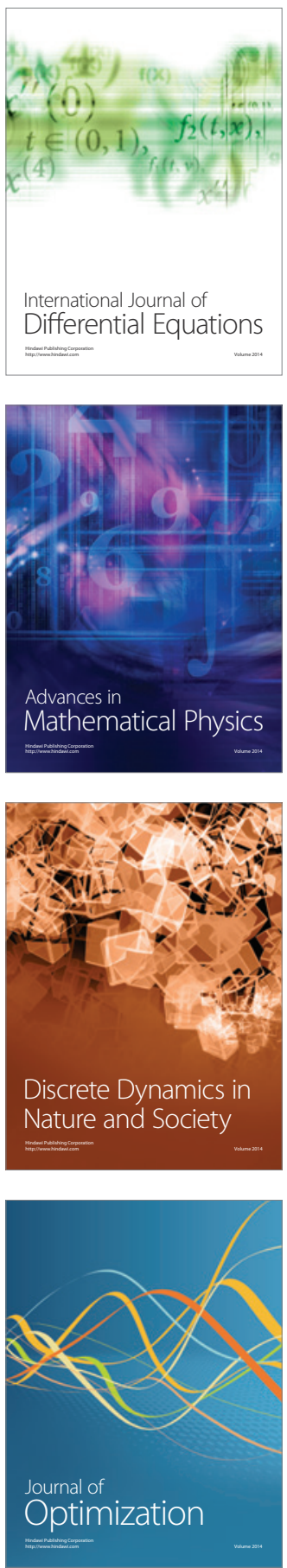